\title{
New insights on the intrinsic, pro-apoptotic effect of IGFB3 in breast cancer
}

\author{
Antonino Belfiore* \\ Endocrinology, Department of Health Sciences, University Magna Graecia of Catanzaro, Catanzaro, Italy \\ *Correspondence: belfiore@unicz.it \\ Edited by: \\ Antimo Migliaccio, Second University of Naples, Italy \\ Reviewed by: \\ Briony Forbes, The University of Adelaide, Australia \\ Paolo Giovanni Vigneri, University of Catania, Italy
}

Keywords: IGFBP3, IGF, cancer vaccines, apoptosis, IGF-I receptor

\section{A commentary on}

Intrinsic, pro-apoptotic effects of IGFBP3 on breast cancer cells are reversible: involvement of PKA, Rho, and ceramide by Perks CM, Burrows C, Holly JMP. Front Endocrinol (2011) 2:29. doi:10.3389/fendo. 2011.00013

Insulin-like growth factor-binding protein 3 (IGFBP3) is the major carrier of circulating IGF-I and IGF-II. In complexes with ALS, IGFBP3 binds IGFs with high affinity and has a pivotal role in modulating IGFs biological effects by limiting their bioavailability. These ternary complexes are stabilized by IGF-I and ALS, both of which are regulated by the pituitary growth hormone. At the tissue level, however, IGFBP3 is subjected to limited proteolysis by several enzymes, which increase the local IGF bioavailability (1). Besides these "IGF-dependent" IGFBP3 effects, which require a functional IGF-IR, several recent studies have provided convincing evidence that IGFBP3 also elicits significant biological effects in an IGFindependent manner. Indeed, by binding a variety of other molecular partners, located both on cell membrane and in the nucleus, IGFBP3 may modulate cell growth, survival, and transformation $(2,3)$. Because of these complex activities, IGFBP3 has been considered both a "gatekeeper," by halting cell proliferation and promoting apoptosis in response to DNA damage, and a "caretaker," by contributing to DNA repair (2).

Studies focusing on the pro-apoptotic and anti-proliferative actions of IGFBP3 have raised hope that these effects could exploited in cancer therapy, but overall conclusions have remained uncertain (35). Even very recent in vivo studies have reached discrepant conclusions. By crossing mice with a genetic deletion of IGFBP3 with mice expressing human $\mathrm{Myc}$ in the prostate, Metha et al. provided in vivo evidence of a role for IGFBP3 as a metastasis suppression gene (6). Accordingly, methylation of the IGFBP3 gene and low IGFBP3 expression occur frequently in aggressive colorectal cancer and are associated with poor response to adjuvant chemotherapy (7). In contrast, in esophageal squamous cell carcinoma, IGFBP3, which is induced by hypoxia, mediates the induction of $\mathrm{CD} 44 \mathrm{H}$ cells by suppressing reactive oxygen species (ROS) (8).

Given the context-dependent, multimodal effects of unliganded IGFBP3, mechanistic studies in well-defined cancer cells are therefore needed. In this respect, the study by Perks et al. (9) is a good step in the right direction. In this study, using Hs578T human breast cancer cells, the authors have dissected a signaling cascade activated by IGFBP3, shedding new light on IGF-independent, complex effects of IGFBP3 on apoptosis. As previously described, this cascade is initiated at the membrane level by IGFBP3 binding to caveolin-1 (Cav-1) (10) at the level of Cav-1 scaffolding domain, which also binds and inactivates protein kinase $\mathrm{A}$ (PKA) (11). Now, the authors show that, upon binding to Cav-1, IGFBP3 induces Hs578T cell apoptosis by preventing PKA inactivation. Indeed, the PKA inhibitor KT5720 was able to revert IGFBP3-induced apoptosis. To better elucidate this pathway, the Authors investigated a possible role of Rho in this pathway. The effects of Rho are regulated by phosphorylation by a specific serine/threonine kinase (ROCK). Notably, the inhibition of ROCK not only blocked the apoptotic effect of IGFBP3, but enabled IGFBP3 to act as a survival factor, confirming an important role for Rho. Using ROCK inhibitors, it was possible to establish that Rho is downstream of the Cav-1/PKA complex. Previously, the same authors had shown that IGFBP3 binds also to beta 1 integrin (10), which now may be considered, together with Cav-1, upstream to the PKA/ROCK/Rho pathway. To add complexity to this model, the authors also showed that this pathway affects the so-called phospholipid rheostat, which regulates the balance between the pro-apoptotic ceramide and the prosurvival sphingosine-1-phosphate (S1P). In fact, Rho was found to positively regulate ceramide production, which in turn was involved in MAPK activation and apoptosis. Indeed, ceramide production is essential to the apoptotic effect of IGFBP3 along this pathway. However, S1P is enhanced by various growth factors including EGF, IGF-1, and insulin, which activate the S1P kinase, SphK1 (12). IGFBP3 itself may stimulate SphK1 and favor S1P synthesis, which in turn may transactivate EGFR and IGF-IR $(13,14)$. Therefore, in conditions in which ceramide synthesis is impaired, IGFBP3 may shift the ceramide to S1P balance in favor of S1P and induce survival. This paper of Perks et al. has the merit of having dissected a major pathway activated by unliganded IGFBP3, which may induce both pro-apoptotic and pro-survival effects in a context-specific manner. 
Several questions still remain open. Firstly, the context-specific variables that affect the balance between these two opposite effects are still largely unknown. The extracellular matrix composition may certainly be a variable through the involvement of integrins. Indeed, the proapoptotic effect of IGFBP3 could be reversed by disrupting integrin receptor complexes (10). The expression level of Cav-1 in cancer cells might also be a variable. It should be mentioned that of Cav-1 is mostly overexpressed during neoplastic progression, and that its role in cancer biology appears to be multidimensional, as it may promote tumorigenesis and metastasis, especially in most advanced cancers, while behaving as an anti-oncogene in other contexts (15). Whether tumor stage may also influence IGFBP3 action is poorly understood.

Secondly, cancer associated fibroblasts (CAFs) may also express dysregulated Cav1 and IGF system components. Interestingly, elevated IGFBP3 has been shown to play a crucial role in fibroblast-tomyofibroblast differentiation in high-grade prostate cancer (16). Moreover, loss of Cav1 in CAFs seems to be a marker of oxidative stress and hypoxia and, in breast cancer, is associated with poor clinical outcome (17). One may wonder how Cav-1 and IGFBP3 interact in CAFs.

A third question to be answered is how the tumor microenvironment may influence IGFBP3 expression, and how the effects of IGFBP3 in CAFs and epithelial cells cooperate.

Finally, the interplay between IGFdependent and IGF-independent effects of IGFBP3 is difficult to address, and is likely to be context-dependent. Although it might be reasonable to expect that a tumor addicted to IGF-IR/IGFs or IRA/IGF-2 circuits may favorably respond to IGF-blocking activity of IGFBP3, it should be considered that high levels of IGFs and IGFBP3 may switch the balance of the phospholipid rheostat in favor of SP1 by activating SphK1. In this context, the concomitant inhibition of SphK1 should perhaps be taken into consideration.
Overall, it appears that understanding the diverse abilities of IGFBP3 is certainly not straightforward, and more mechanistic studies, possibly on breast cancer stem cells, are needed to establish whether IGFBP3 could be exploited in breast cancer therapy.

\section{ACKNOWLEDGMENTS}

This work was supported in part by grants from the Associazione Italiana per la Ricerca sul Cancro (AIRC) (grant $n$. 14066/13) to Antonino Belfiore.

\section{REFERENCES}

1. Jones JI, Clemmons DR. Insulin-like growth factors and their binding proteins: biological actions. Endocr Rev (1995) 16:3-34. doi:10.1210/ edrv-16-1-3

2. Baxter RC. Insulin-like growth factor binding protein-3 (IGFBP-3): novel ligands mediate unexpected functions. J Cell Commun Signal (2013) 7:179-89. doi:10.1007/s12079-013-0203-9

3. Jogie-Brahim S, Feldman D, Oh Y. Unraveling insulin-like growth factor binding protein3 actions in human disease. Endocr Rev (2009) 30:417-37. doi:10.1210/er.2008-0028

4. Perks C, Holly J. Actions of IGFBP on epithelial cancer cells: potential for new therapeutic targets. Horm Metab Res (2003) 35:828-35. doi:10.1055/s2004-814139

5. Baxter RC. IGF binding proteins in cancer: mechanistic and clinical insights. Nat Rev Cancer (2014) 14:329-41. doi:10.1038/nrc3720

6. Mehta HH, Gao Q, Galet C, Paharkova V, Wan J, Said J, et al. IGFBP-3 is a metastasis suppression gene in prostate cancer. Cancer Res (2011) 71:5154-63. doi:10.1158/0008-5472.CAN10-4513

7. Perez-Carbonell L, Balaguer F, Toiyama Y, Egoavil C, Rojas E, Guarinos C, et al. IGFBP3 methylation is a novel diagnostic and predictive biomarker in colorectal cancer. PLoS One (2014) 9:e104285. doi:10.1371/journal.pone.0104285

8. Natsuizaka M, Kinugasa H, Kagawa S, Whelan KA, Naganuma S, Subramanian H, et al. IGFBP3 promotes esophageal cancer growth by suppressing oxidative stress in hypoxic tumor microenvironment. Am J Cancer Res (2014) 4:29-41.

9. Perks CM, Burrows C, Holly JMP. Intrinsic, proapoptotic effects of IGFBP-3 on breast cancer cells are reversible: involvement of PKA, Rho, and ceramide. Front Endocrinol (Lausanne) (2011) 2:13. doi:10.3389/fendo.2011.00013

10. Burrows C, Holly JMP, Laurence NJ, Vernon EG, Carter JV, Clark MA, et al. Insulin-like growth factor binding protein 3 has opposing actions on malignant and nonmalignant breast epithelial cells that are each reversible and dependent upon cholesterol-stabilized integrin receptor complexes. Endocrinology (2006) 147:3484-500. doi:10.1210/ en.2006-0005
11. Levin AM, Murase K, Jackson PJ, Flinspach ML, Poulos TL, Weiss GA. Double barrel shotgun scanning of the caveolin-1 scaffolding domain. ACS Chem Biol (2007) 2:493-500. doi:10.1021/ cb700055t

12. Pyne NJ, Tonelli F, Lim KG, Long JS, Edwards J, Pyne S. Sphingosine 1-phosphate signalling in cancer. Biochem Soc Trans (2012) 40:94-100. doi:10. 1042/BST20110602

13. Martin JL, Lin MZ, McGowan EM, Baxter RC. Potentiation of growth factor signaling by insulinlike growth factor-binding protein-3 in breast epithelial cells requires sphingosine kinase activity. J Biol Chem (2009) 284:25542-52. doi:10.1074/jbc. M109.007120

14. De Mellow JS, Baxter RC. Growth hormonedependent insulin-like growth factor (IGF) binding protein both inhibits and potentiates IGF-I-stimulated DNA synthesis in human skin fibroblasts. Biochem Biophys Res Commun (1988) 156:199-204. doi:10.1016/S0006-291X(88) 80824-6

15. Patani N, Martin L-A, Reis-Filho JS, Dowsett M. The role of caveolin-1 in human breast cancer. Breast Cancer Res Treat (2012) 131:1-15. doi:10. 1007/s10549-011-1751-4

16. Sampson N, Zenzmaier C, Heitz M, Hermann M, Plas E, Schäfer G, et al. Stromal insulin-like growth factor binding protein 3 (IGFBP3) is elevated in the diseased human prostate and promotes ex vivo fibroblast-to-myofibroblast differentiation. Endocrinology (2013) 154:2586-99. doi: 10.1210/en.2012-2259

17. Sotgia F, Martinez-Outschoorn UE, Pavlides S, Howell A, Pestell RG, Lisanti MP. Understanding the Warburg effect and the prognostic value of stromal caveolin-1 as a marker of a lethal tumor microenvironment. Breast Cancer Res (2011) 13:213. doi: $10.1186 /$ bcr2892

Conflict of Interest Statement: The author declares that the research was conducted in the absence of any commercial or financial relationships that could be construed as a potential conflict of interest.

Received: 02 September 2014; paper pending published: 24 September 2014; accepted: 05 October 2014; published online: 21 October 2014.

Citation: Belfiore A (2014) New insights on the intrinsic, pro-apoptotic effect of IGFB3 in breast cancer. Front. Endocrinol. 5:176. doi: 10.3389/fendo.2014.00176

This article was submitted to the journal Frontiers in Endocrinology.

Copyright (c) 2014 Belfiore. This is an open-access article distributed under the terms of the Creative Commons Attribution License (CC BY). The use, distribution or reproduction in other forums is permitted, provided the original author(s) or licensor are credited and that the original publication in this journal is cited, in accordance with accepted academic practice. No use, distribution or reproduction is permitted which does not comply with these terms. 